\title{
SPC without Control Limits and Normality Assumption: A New Method
}

\author{
J.A. Vazquez-Lopez and I. Lopez-Juarez ${ }^{\star}$ \\ Instituto Tecnologico de Celaya \\ Centro de Investigacion y de Estudios Avanzados del IPN - Unidad Saltillo \\ ismael.lopez@cinvestav.edu.mx
}

\begin{abstract}
Control Charts (CC) are important Statistic Process Control (SPC) tools developed in the 20's to control and improve the quality of industrial production. The use of $\mathrm{CC}$ requires visual inspection and human judgement to diagnoses the process quality properly. CC assume normal distribution in the observed variables to establish the control limits. However, this is a requirement difficult to meet in practice since skewness distributions are commonly observed. In this research, a novel method that neither requires control limits nor data normality is presented. The core of the method is based on the FuzzyARTMAP (FAM) Artificial Neural Network (ANN) that learns special and non-special patterns of variation and whose internal parameters are determined through experimental design to increase its efficiency. The proposed method was implemented successfully in a manufacturing process determining the statistical control state that validate our method.
\end{abstract}

Keywords: Control Charts, Neural Networks, Pattern Recognition.

\section{Introduction}

In manufacturing processes, the use of Control Charts (CC) is a common technique used to monitor the quality of the production. Variables are monitored to preserve the process under statistical control and also to detect any special variation. Should this situation occurs, then specially trained personnel take the appropriate actions to get the process back into control. By using CC it is possible to know when the process presents a special behaviour by monitoring its upper and lower control limits. However, using this approach, it is not possible to determine the type of pattern. To overcome this limitation, a novel method to recognise and analyse statistical quality patterns using the Fuzzy ARTMAP (FAM) Artificial Neural Network (ANN) is proposed. The FAM network parameters are determined off-line using experimental design and the Monte Carlo method which constitutes a novel method to increase the FAM efficiency eliminating the trial and error procedure commonly used [11]. During testing, the FAM Learning parameters are selected automatically depending if special or non-special pattern is encountered. The system is able to recognise both pattern types such as non special: natural in control; and special: upward shift,

\footnotetext{
^ Corresponding author.
} 
downward shift, upward trend and downward trend. In order to improve the discrimination new patterns can be added to the Initial Knowledge Base (IKB) forming what it is referred to as the Enhanced Knowledge Base (EKB). The network is retrained on-line to take into account the new pattern information which improves the pattern recognition capability of the system. The proposed method consists of two modules, the learning process and the control process. The first module includes basically the IKB and the EKB whereas the second module includes the pattern recognition and the control stages.

The use of this new method also begins a new Statistical Process Control (SPC) methodology since data normality is not required in the probability distribution of manufacturing processes as it was required in earlier production systems (not automated) using CC.

The rest of this article is organised as follows. In section 2 , background information in terms of related work done by other researchers is presented while in section 3, the Fuzzy ARTMAP neural network is described in detail. Section 4 formally presents and describes the developed method. Results are given in section 5 and finally conclusions are provided and further work envisaged in section 6 .

\section{Related Work and Original Contribution}

Considering the disadvantages of the $\mathrm{CC}$, diverse investigations suggest the use of

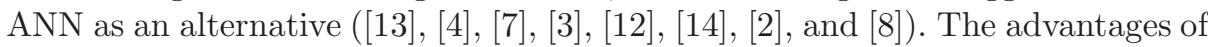
using ANN's in comparison with CC are: a) It is possible to work in real-time 5 . b) The assumption of data normality is not necessary [9]; and c), great amounts of complex data can be processed in a short time [10. Hindi, used the Fuzzy ARTMAP to determine the type of change presented in the process parameters 6]. He compares the results with the obtained from the application of the $\bar{X}$ and R-chart. He used values 0 and 3 for $\mu$ and 1 and 3 for $\sigma$, considering the combination $\mu=0$ and $\sigma=1$ to represent a state of statistical control. The FAM parameter values were fixed. Guh, proposed the use of ANN Back-Propagation $(\mathrm{BPN})$ in combination with a decision tree for pattern recognition [5]. In his work, Guh makes reference to three modules. Module A is in charge of data preprocessing, module $\mathrm{B}$ works like a $\mathrm{CC}$ detecting abnormal cases of variation whereas the module $\mathrm{C}$ determines the type of pattern based on a pre-defined decision tree. Our method compares favourably to previous work having the following advantages:

- Network parameters are determined through experimental design for maximum efficiency.

- Normality assumption is not required.

- High sampling size to guarantee data normality is not required.

- ANN testing parameters are selected automatically according to the type of probability distribution.

- The model refines its knowledge through real-world data. 


\section{FuzzyARTMAP (FAM)}

The FuzzyARTMAP neural network is based on the Adaptive Resonance Theory (ART) which was developed by Stephen Grossberg and Gail Carpenter at Boston University. In Fuzzy ARTMAP there are two modules $A R T_{a}$ and $A R T_{b}$ and an inter-ART module "map field" that controls the learning of an associative map from $A R T_{a}$ recognition categories to $A R T_{b}$ recognition categories [1]. The FAM architecture is shown in figure 1.

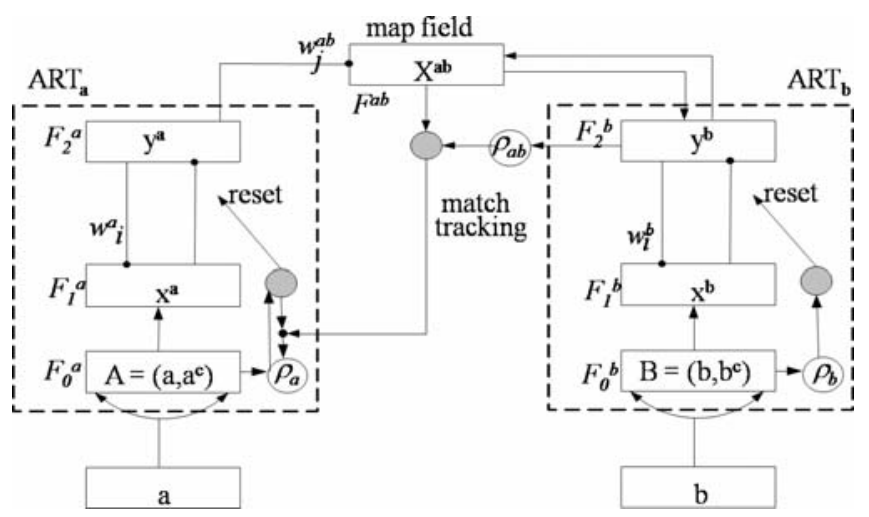

Fig. 1. FuzzyARTMAP Architecture

The map field module also controls the match tracking of $A R T_{a}$ vigilance parameter. A mismatch between Map field and $A R T_{a}$ category activated by input $\mathbf{a}$ and $A R T_{b}$ category activated by input $\mathbf{b}$ increases $A R T_{a}$ vigilance by the minimum amount needed for the system to search for, and if necessary, learn a new $A R T_{a}$ category whose prediction matches the $A R T_{b}$ category. The search initiated by the inter-ART reset can shift attention to a novel cluster of features that can be incorporated through learning into a new $A R T_{a}$ recognition category, which can then be linked to a new ART prediction via associative learning at the Map field. The algorithm uses a preprocessing step, called complement coding which is designed to avoid category proliferation. Similar to ART-1, a vigilance parameter measures the difference allowed between the input data and the stored pattern. Therefore this parameter is determinant to affect the selectivity or granularity of the network prediction. For learning, the FuzzyARTMAP has 4 important factors: Vigilance in the input module $\left(\rho_{a}\right)$, vigilance in the output module $\left(\rho_{b}\right)$, vigilance in the Map field $\left(\rho_{a b}\right)$ and learning rate $(\beta)$. These were the considered factors in this research. The FAM algorithm was coded in $\mathrm{C}++$ using the Visual Studio 2005 compiler running in a Core2Duo PC computer at $1.86 \mathrm{GHz}$. 


\section{Method}

The proposed method consists of three important elements: Learning process, Control process and the use of the EKB as shown in figure 2. The first element contains tree sub-elements; the random variable from groups of 20 data point 1 and the Fuzzy ARTMAP learning using either the IKB or the IKB + EKB.

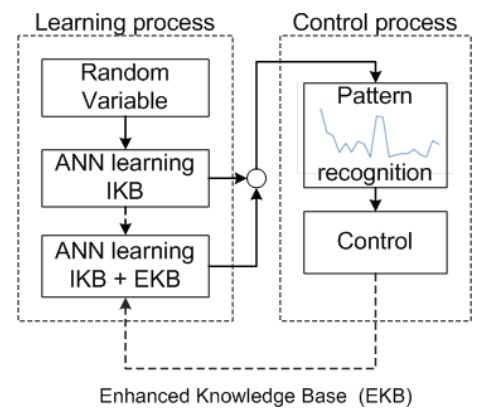

Fig. 2. General Method

Initially the FuzzyARTMAP network is trained with an IKB that was obtained from the Monte Carlo simulation. The data considered special and non special patterns as defined by the following equation: $X_{t}=\mu+n_{t}+d_{t}$ where $\mu$ is the effect of the global data (mean), $X_{t}, n_{t}$ and $d_{t}$ are the the data, the effect of the natural variation and the effect of the special variation in time $t$, respectively [5]. A sample data with a natural pattern has $d_{t}=0$, if it is unnatural, then $d_{t}>0$. The Control process is formed by the pattern recognition and the control stages, which are better explained using figure 3. Finally, the EKB serves to add unknown patterns to the ANN learning.

\subsection{Pattern Recognition}

Figure 3 shows the algorithm to maintain the process under statistical control. The algorithm uses two stages, the pattern recognition and control. During pattern recognition the $\mathrm{X}$ vector is preprocessed as follows:

Standardization. The standardization of the $\mathrm{X}$ vector is obtained by $Y=$ $(X-\varepsilon) / \tau$. where $\varepsilon$ and $\tau$ are objective values for de mean and the standard deviation respectively. The real mean and standard deviation of $X$ vector are $\bar{X}$ and $S$, respectively. If $\bar{X} \rightarrow \varepsilon$, and $S \rightarrow \tau$, then the $X$ vector will be a non special pattern. Otherwise, the $\mathrm{X}$ vector will be a special pattern and the pattern has to be identified among the upward or downward shift, upward or downward trend or any other possible special pattern. The other special patterns can be cyclical, systematic or mixture. These other special patterns were not considered in this

\footnotetext{
${ }^{1}$ Using this group size an efficiency higher than $90 \%$ was obtained during experiments.
} 


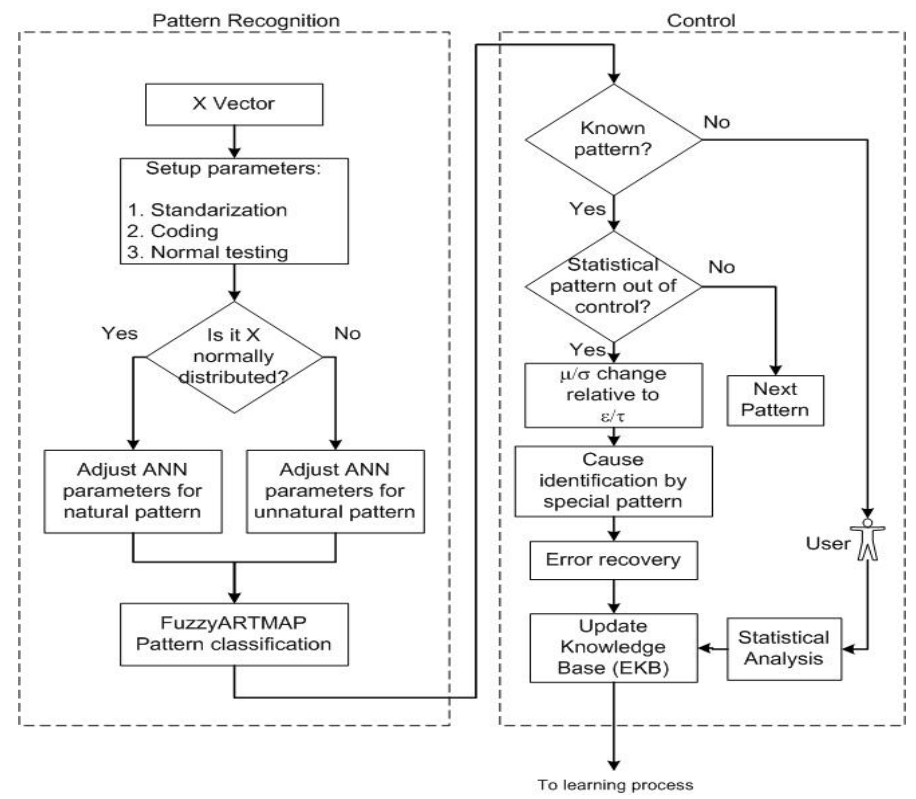

Fig. 3. Process Control Method

paper since they are more dependent from the real process and its inclusion is considered in the EKB. All special pattern data cannot have only non normal distribution and this is the reason to group them in one IKB. Another IKB was integrated by only the $X$ vector with normal distribution, but, is possible to find special and non special pattern data in it. For example, if $\bar{X}$ or $S$ are near to $\varepsilon$ and $\tau$, but not sufficiently, the $X$ vector can be special pattern data with normal distribution and natural pattern too. If $a$ is the absolute difference between $\varepsilon$ and $\bar{X}$ or between $\tau$ and $S$, then, there exists a numeric value given by $a$ so that the probability distribution of $X$ is not normal.

Coding. The coding stage consist of transforming the $\mathrm{X}$ vector within the range $[0,1]$ using a lineal transformation.

Test for normal distribution This test is important since it indicates the type of FuzzyARTMAP learning parameters to be used depending if the data set is normally distributed or not. After selecting the proper ANN parameters the learning starts by training the FuzzyARTMAP network using the IKB as a training set which establishes the internal representation of the network.

\subsection{Control}

Once the statistical pattern is recognised, the control stage begins. If the pattern is not recognised by the FAM network, then user intervention is required to statistically analyze and classify the pattern. On the other hand the pattern can 
be classified as belonging to process in control or process out of control. A pattern belonging to a process in control indicates stability in the manufacturing process. In this case, a new $\mathrm{X}$ vector will be presented. Otherwise, the manufacture process is out control, then the special pattern is identified and a proper error recovery strategy implemented. When a special pattern occurs the first time, this pattern will be different from the simulated patterns used to train the FAM and it contains real data from the process so this information is used to enhance the previous knowledge base (IKB) forming the new EKB. In practice, this resulted in a fine tuning mechanism to increase the expertise and robustness of the neural network as demonstrated by the obtained results in a real process as indicated in the following section.

\section{$5 \quad$ Issues and Experiments}

The FuzzyARTMAP learning parameters considered for the experimental work are: the input base vigilance $\left(\rho_{a_{1,2}}\right)$, vigilance in the map-field $\left(\rho_{a b_{1,2}}\right)$, learning rate $\left(\beta_{1,2}\right)$ and the output base vigilance $\left(\rho_{b_{1,2}}\right)$.

After trials with different parameters, a factorial fractional design $\left(2_{I I I}^{k}\right)$, with 7 factors, 2 replicates, 16 experimental runs and a significance level of 0.05 resulted appropriate. The values for the factors levels were 0.2 and 0.8 for all them, with the exception of $\beta_{1,2}$ where the high level was set to 1.0. This experimentation employed the Monte Carlo Simulation method to generate the train and test set of vectors with special and non special patterns. X Vectors with non special natural pattern (a), special natural pattern (b), and special unnatural pattern (c) were considered during the experiments. Cases (a) and (b) have normal distribution and (c) non normal. For case (a), it was considered $\mu=0$ and $\sigma=1$; case (b) considered the combination $(\mu, \sigma)$ with values $(0,3),(3,1)$ and $(3,3)$. Finally, for the third case (c), the special patterns were obtained varying the term $d$ in equation (1). The upward shift pattern was generated with $d>2.5 \sigma$, the downward shift was generated with $d<-2.5 \sigma$. The upward trend pattern was generated with $d>0.1 \sigma$ and for the downward shift $d<-0.1 \sigma$. A detailed explanation of the simulation and the selection of the network parameters it is given in our previous work [1]. The best parameters values determined after the experimental design are shown in the Table 1. These values are adjusted automatically during the training phase of the FuzzyARTMAP network depending on the type of detected pattern.

The validation of our method was carried out using simulated and real-world data3. The data presented in this paper comes from a make-up manufacturing company representing the level of quality conformance during the product packing stage. The process generated values which are the fraction of non-conform products ( $X$ vector). In the method, considering the objective non-conform fraction $(p=0.005)$ resulted in $\varepsilon=0.69$ and $\tau=0.83$. The number of $\mathrm{X}$ vectors processed by the algorithm are 21 . The results are given in table 2

\footnotetext{
${ }^{2}$ Subindexes 1,2 indicate parameters used during training/testing phase, respectively.
}

${ }^{3}$ Due to space limitation, only results from one industrial process are presented. 
Table 1. FuzzyARTMAP parameters

\begin{tabular}{|c|c|c|}
\hline $\begin{array}{c}\text { ANN } \\
\text { Parameter }\end{array}$ & $\begin{array}{c}\text { Normal Dist. } \\
\text { Pattern }\end{array}$ & $\begin{array}{c}\text { Nonnormal Dist. } \\
\text { Pattern }\end{array}$ \\
\hline$\rho_{a_{1}}$ & 0.2 & 0.2 \\
$\rho_{a_{2}}$ & 0.2 & 0.2 \\
$\rho_{b_{1,2}}$ & 0.9 & $>0.7$ \\
$\rho_{a b_{1}}$ & any & 0.2 \\
$\rho_{a b_{2}}$ & any & 0.8 \\
$\beta_{1}$ & 1.0 & 0.2 \\
$\beta_{2}$ & 1.0 & 1.0 \\
\hline
\end{tabular}

Table 2. Results form the packing process

\begin{tabular}{|c|l|l|l|l|}
\hline Vector & Distribution & Process & \multicolumn{1}{|c|}{ Results $(C C)$} & Results $(A N N)$ \\
\hline 1 & Non normal & Out control & Out control-downward trend & Downward trend \\
2 to 6 & Non normal & Out control & In control & Downward trend \\
7 & Non normal & Out control & Out control-downward trend & Downward trend \\
8 & Non normal & Out control & In control & Downward trend \\
9 & Non normal & Out control & Out control-downward trend & Downward trend \\
10,11 & Non normal & Out control & In control & Downward trend \\
12 to 19 & Normal & In control & In control & Natural In control \\
20 & Non normal & Out control & Out control-downward trend & Downward trend \\
21 & Non normal & Out control & Out control & Downward trend \\
\hline
\end{tabular}

\section{Conclusions and Ongoing Work}

An alternative method for Statistical Proces Control (SPC) that does not require control limits, assumption of normality in the process data and high simple size (to secure data normality) was presented.

It was observed that grouping family vectors according to its probability distribution (normal or non normal) and using two sets of network training parameters increased the neural network efficiency. The method was tested during simulation and also with process data from a make-up company comparing the obtained results with the use of Control Charts (CC). The proposed method compared favourably considering that it can be applied to either continuous or discrete variables, The efficiency of the method is improved when unknown patterns are added to the Initial Knowledge Base using the incremental learning properties of the FuzzyARTMAP network. The method is intended to be applied on-line to automatically monitor the quality of the production, therefore on going work is looking at the implementation in other industrial sectors.

Acknowledgments. The authors wish to thank CONACyT for the financial support given to the project through grant 61373 and also for the scholarship granted to J. A. Vazquez-Lopez during his doctoral studies. 


\section{References}

1. Carpenter, G.A., Grossberg, S., Markuzon, N., Reynolds, J.H., Rosen, D.B.: FuzzyARTMAP: A neural network architecture for incremental learning of analog multidimensional maps. IEEE Trans. on Neural Networks 3(5), 698-713 (1992)

2. Cheng, C.S.: A neural network approach for the analysis of control chart patterns. International Journal of Production Research 35(3), 667-697 (1997)

3. Guh, R.S., Tannock, J.D.T.: Recognition of control chart concurrent patterns using a neural network approach. Int. J. Prod. Res. 37(8), 1743-1765 (1999)

4. Guh, R.S.: Robustness of the neural network based control chart pattern recognition system to non-normality. Int. J. Qual. Reliability Mgmt 19(1), 97-112 (2002)

5. Guh, R.S.: Real-time pattern recognition in statistical process control: a hybrid neural network/decision tree-based approach. IMechE. J. Engineering Manufacture 219, Part B (2005)

6. Al-Hindi, H.A.: Control Chart Interpretation Using Fuzzy ARTMAP. Journal of King Saud University. Engineering Sciences 16(1) (2004)

7. Ho, E.S., Chang, S.I.: An integrated neural network approach for simultaneous monitoring of process mean and variance shifts - a comparative study. Int. J. Prod. Res. 37(8), 1881-1901 (1999)

8. Hwarng, H.B., Chong, C.W.: Detecting process nonrandomness through a fast and cumulative learning ART-based pattern recognizer. Int. J. Prod. Res. 33(7), 18171833 (1995)

9. Pacella, M., Semeraro, Q., Anglania, A.: Manufacturing quality control by means of a Fuzzy ART network trained on natural process data. Engineering Applications of Artificial Intelligence 17, 83-96 (2004)

10. Pacella, M., Semeraro, Q.: Understanding ART-based neural algorithms as statistical tools for manufacturing process quality control. Engineering Applications of Artificial Intelligence 18, 645-662 (2005)

11. Vazquez-Lopez, J.A., Lopez-Juarez, I., Peña-Cabrera, M.: Experimental Design applied to the FuzzyARTMAP neural network to the statistical special pattern recognition. In: XVIII Congress of the Automatic Control Chilean AssociationIFAC-IEEE (2008) (in spanish)

12. Wani, M.A., Pham, D.T.: Efficient control chart pattern recognition through synergistic and distributed artificial neural networks. Proc. Instn Mech. Engrs, Part B: J. Engineering Manufacture 213(B2), 157-169 (1999)

13. Zobel, C.W., Cook, D.F., Nottingham, Q.J.: An augmented neural network classification approach to detecting mean shifts in correlated manufacturing process parameters. International Journal of Production Research 42(4), 741-758 (2004)

14. Zorriassantine, F., Tannock, J.D.T.: A review of neural networks for statistical process control. Journal of Intelligent Manufacturing 9, 209-224 (1998) 